PREPARED FOR SUBMISSION TO JCAP

\title{
Where the world stands still: turnaround as a strong test of $\Lambda \mathrm{CDM}$ cosmology
}

\author{
V. Pavlidou ${ }^{a, b}$ T. N. Tomaras ${ }^{a}$ \\ ${ }^{a}$ Department of Physics and ITCP ${ }^{1}$, University of Crete, University of Crete, 71003 Herak- \\ lion, Greece \\ ${ }^{b}$ Foundation for Research and Technology - Hellas, IESL, Voutes, 71110 Heraklion, Greece \\ E-mail: pavlidou@physics.uoc.gr, tomaras@physics.uoc.gr
}

\begin{abstract}
Our intuitive understanding of cosmic structure formation works best in scales small enough so that isolated, bound, relaxed gravitating systems are no longer adjusting their radius; and large enough so that space and matter follow the average expansion of the Universe. Yet one of the most robust predictions of $\Lambda \mathrm{CDM}$ cosmology concerns the scale that separates these limits: the turnaround radius, which is the non-expanding shell furthest away from the center of a bound structure. We show that the maximum possible value of the turnaround radius within the framework of the $\Lambda \mathrm{CDM}$ model is, for a given mass $M$, equal to $\left(3 G M / \Lambda c^{2}\right)^{1 / 3}$, with $G$ Newton's constant and $c$ the speed of light, independently of cosmic epoch, exact nature of dark matter, or baryonic effects. We discuss the possible use of this prediction as an observational test for $\Lambda \mathrm{CDM}$ cosmology. Current data appear to favor $\Lambda \mathrm{CDM}$ over alternatives with local inhomogeneities and no $\Lambda$. However there exist several local-universe structures that have, within errors, reached their limiting size. With improved determinations of their turnaround radii and the enclosed mass, these objects may challenge the limit and $\Lambda \mathrm{CDM}$ cosmology.
\end{abstract}

\footnotetext{
${ }^{1}$ Institute of Theoretical and Computational Physics (formerly Institute of Plasma Physics)
} 


\section{Contents}

1 Introduction 1

2 Qualitative considerations: why does the turnaround radius have a bound? 3

3 Quantitative derivations of the turnaround radius bound 4

3.1 Spherical mass in de Sitter space 4

3.2 Spherical collapse of early-Universe perturbation 5

3.3 Zero Acceleration Mass Shell 5

4 Testing the bound observationally $\quad 6$

$\begin{array}{lll}4.1 & \text { What observations are necessary? } & 7\end{array}$

4.2 How many structures need to be observed? 8

4.3 Which structures should we be observing? $\quad 8$

4.4 Can such observations produce "false positives"? 9

4.5 How accurate need the observations be? 9

$\begin{array}{ll}\text { 4.6 What can already existing data tell us? } & 10\end{array}$

5 Discussion

\section{Introduction}

In spite of great successes of the $\Lambda \mathrm{CDM}$ model in the interpretation of cosmological observations, including the cosmic microwave background, supernovae type Ia, and the large scale structure in the universe, alternative cosmologies continue to attract interest and cause intense debate. At the heart of the issue lies the -unknown- physics of the dark sector.

Weakly interacting particle dark matter has by now accumulated astrophysical evidence that goes beyond its "relic abundance miracle" appeal (e.g. [1, 2]). For example, combined imaging of merging clusters both through the X-ray emission of shocked gas (tracing the collisional baryonic component) and through lensing (tracing the gravitational potential peaks), have shown a clear separation between the two and very different geometries between them [3-5]. However, the up to now lack of any sign for TeV-scale supersymmetry or an indisputable signature of any of the prime candidates for particle dark matter ${ }^{1}$ has intensified the analysis of alternative proposals as resolutions of the "dark matter" puzzle, with Modified Newtonian Dynamics (MOND) being the most popular among them (e.g., $[9,10])$, although attempts to reconcile the observations of merging clusters with MOND require the existence of at least some particle dark matter, such as sterile neutrinos [11].

Our current best estimates on the other component of the dark sector, dark energy [12], indicate that it behaves effectively as a cosmological constant, but with an uncomfortably low value, defying both microscopic and "anthropic" interpretations [13].

Without a confirmed microphysical foundation, $\Lambda \mathrm{CDM}$ is therefore our current-best effective theory of the evolution of the Universe. Observations that might contradict its predictions

\footnotetext{
${ }^{1}$ despite several potentially interesting signals (e.g., most recently, the Galactic Center gamma-ray signal [6] and the X-ray signal from galaxy clusters $[7,8])$
} 
and illuminate the direction towards the necessary new physics to complete our understanding of the cosmos are thus actively pursued. However, these are frequently hindered by unavoidable complexities in connecting theory with the real world. For example, there is persisting tension between observations and predictions of the cold dark matter (CDM) model for relatively small scales, such as the abundance of dwarf galaxies and the central profiles of dark matter halos (e.g. [14]). At these scales though, the (much more complex and difficult to simulate) behavior of luminous matter plays a dominant role in determining both the shape of central profiles and the observability of small satellite galaxies (e.g., [15, 16]; see however [17] for counter-arguments on the efficacy of baryonic physics in resolving the problems of CDM on small scales); in addition, the possibility of more complex dark matter physics (decays, self-interactions, warm/mixed dark matter, e.g. [18-22]) further complicate the comparison between theory predictions and observations.

Additional complications in comparing the results of $\Lambda \mathrm{CDM}$ simulations to observations arise due to a certain vagueness on where one should place the boundary of a bound, virialized dark matter halo in the CDM model. Simulated CDM halos are fitted well (at least on scales of interest for defining their outer radius) by the Navarro-Frenk-White density profile [23], which however does not have a clear outer boundary. Rather, it follows an $r^{-3}$ power-law profile at large radii, with the mass included within $r$ diverging logarithmically as $r \rightarrow \infty$. The usual solution is to take the virial radius to be located where the average density within that radius is a predetermined factor over the background matter density at the redshift of virialization. This idea comes from the spherical collapse model: if one starts with a spherical perturbation in an otherwise homogeneous $\Lambda$ CDM universe and allows it to evolve as a closed universe according to its own Friedmann equation, then one can predict the time it would formally collapse to a singularity, and hence the background matter density at that time. If one additionally assumes that, at this moment of final collapse, the radius of the perturbation, instead of becoming zero, is stabilized at the value predicted by the virial theorem, then one can also calculate the mean density of the perturbation at that time. The ratio of the two densities has a well-defined value, always equal to $18 \pi^{2}$ in an $\Omega_{m}=1$ universe, or, in an $\Omega_{m}+\Omega_{\Lambda}=1$ universe, slowly increasing from $18 \pi^{2}$ at early times (before vacuum energy significantly affects the evolution of the expansion rate) to a value of $\sim 360$ at the present cosmic epoch [24].

This approach involves two important ad hoc assumptions: that the time of virialization of a structure is identical to the time of its formal collapse to a singularity; and that the virial radius as calculated by the recipe above is indeed a meaningful scale for a realistic bound structure in the $\Lambda \mathrm{CDM}$ universe. Neither is obviously true. On the one hand, virialization is a complex process that involves interactions between dark matter particles, as well as between dark matter substructures. The collapse-to-a-singularity timescale is the only calculable timescale in the spherical collapse model so it is the first-recourse choice, but virialization itself cannot be described by the spherical collapse model, and it does not have to operate on its timescale. At best, the time of true virialization can only be associated with the collapse-to-singularity time within a factor of a few. On the other hand, cosmological simulations show that simulated dark matter halos appear themselves unaware of their own virial radius: their radial density profile slope is unchanged below and above the virial radius as calculated above, and no break appears at radii a factor of a few larger or smaller than the virial radius either [23, 25]. In addition, the "virial" radius predicted by the spherical-collapse recipe does not correspond to the spatial extent of relaxed particles [26]. Observational constraints on the extent of bound matter on galactic scales similarly show discrepancies with the usual definitions of the virial radius [27]. 
Given the considerations above, the question then arises: are there any predictions of $\Lambda \mathrm{CDM}$ regarding cosmic structure that are clean of baryonic physics and robust against the details of the way the predicted quantities are defined or calculated?

In this work, we argue that such a prediction does exist, and it involves the turnaround radius of a bound structure - the radius separating infalling or virialized material from the outwards expansion of the Hubble flow. In contrast to the virial radius, this is a well-defined structure boundary in simulations, analytic calculations, and the observable Universe: if we calculate the spherically averaged radial velocity of matter $\left\langle v_{r}\right\rangle$ around a center of gravity, starting from spheres of very large radius and proceeding towards smaller scales, the turnaround radius corresponds to the first time where $\left\langle v_{r}\right\rangle$ becomes zero.

Here we show that the well-established result of $\Lambda \mathrm{CDM}$ structure formation that growth of structure does not continue indefinitely (e.g., [24, 28-35]) can be cast as a robust testable prediction: that the turnaround radius can never exceed an absolute upper bound, which, in an $\Omega_{m}+\Omega_{\Lambda}=1$ universe, is independent of time, and only depends on its mass and the value of the cosmological constant. This bound corresponds to the distance from the center of a bound structure where the influence of matter and dark energy are at a balance. If the mass shell at this radius has not reached turnaround yet, it never will, and neither will any mass shells at larger radii. The universe outside that bound will eventually accelerate away, while the universe as observed by inhabitants of the structure will asymptotically approach the Einstein static universe.

The bound can be tested observationally by estimating independently the turnaround radius and enclosed mass of various structures. The predictions of many alternative to $\Lambda$ CDM cosmological models for the same bound (if one exists at all) are diverse enough that the bound can be used as an important cosmology discriminator. Such observations do exist for a few objects, demonstrating the feasibility of such an endeavor. However, because the bound has not been recognized as a testable cosmological prediction up to now, these observations have not been systematically pursued. We therefore urge for the undertaking of such observational efforts.

\section{Qualitative considerations: why does the turnaround radius have a bound?}

Structure formation in $\Lambda \mathrm{CDM}$ cosmology is a cosmic battle between expansion momentum, gravitational attraction, and dark energy. On small enough scales, isolated, bound, relaxed gravitating systems are no longer adjusting their radius ${ }^{2}$; on very large scales, space and matter follow the average expansion of the Universe. The scales in between, however, warrant more careful consideration [37].

Collapsed structures grow by accreting surrounding mass shells: the gravitational attraction of the central object will slow down a larger, co-centric mass shell and detach it from the Hubble flow, causing it to turn around and eventually collapse onto the central object. In a $\Lambda$ CDM universe, however, this process can only operate as long as the effect of dark energy inside the mass shell does not overcome that of matter. The dark energy associated with a cosmological constant does not change with the scale factor $a_{p}$ of the perturbation, while matter density falls as $a_{p}^{-3}$ : as the scale factor of a mass shell grows, the effect of dark energy within it grows relative to that of matter. If the shell fails to reach turnaround by the time the two effects

\footnotetext{
${ }^{2}$ Before the effect of $\Lambda$ quenches structure growth, the mass of non-isolated cosmic structures changes through accretion and minor and major mergers; see e.g. [36] for a recent discussion
} 
become equal, dark energy will win, and the shell will start expanding at an accelerated rate. The mass $M$ enclosed by the last shell that manages to reach turnaround will be the final mass of that particular bound structure. The radius at turnaround of this last shell is the maximum turnaround radius, $R_{\mathrm{ta} \text {,max }}$, of mass $M$. The universe outside $R_{\mathrm{ta} \text {,max }}$ will eventually accelerate away, while the universe as observed by inhabitants of the structure inside $R_{\mathrm{ta} \text {,max }}$ will asymptotically approach the Einstein static universe. The last shell to turnaround will grow increasingly slowly and will only reach its final size, $R_{\mathrm{ta} \text {,max }}$, after an infinite time.

In the following section we will derive the relation between $R_{\mathrm{ta}, \max }$ and $M$.

\section{Quantitative derivations of the turnaround radius bound}

The value of the bound is robust against the detailed assumptions of its calculations, as we demonstrate by following through three different lines of derivation. First, we consider the motion of a test particle under the influence of a point mass in de Sitter space (in which case the mass and $\Lambda$ are the only ingredients of the universe) and calculate the conditions under which, when left from rest, the particle will fall toward the mass, or it will be dragged away by the influence of $\Lambda$. Next, we follow the evolution of a spherical density perturbation within an otherwise homogeneous and isotropic universe of $\Omega_{m}+\Omega_{\Lambda}=1$, from very early times when it co-expands with the background universe, until the moment of turnaround (the usual setup for the spherical collapse model, where homogeneity is assumed both for the evolving perturbation as well as for the background universe). Finally, we use the time derivative of the Friedmann equation to seek a point of (unstable) equilibrium, with not only zero expansion rate but also zero acceleration.

\subsection{Spherical mass in de Sitter space}

The spacetime outside a spherically symmetric matter distribution with mass $M$ in a spatially flat de Sitter background with cosmological constant $\Lambda>0$ is described by the Schwarzschild de Sitter (SdS) metric [38-40], which in static coordinates is $d \tau^{2}=A(r) d t^{2}-d r^{2} / A(r)-r^{2} d \Omega^{2}$, with $A(r)=1-2 M / r-\Lambda r^{2} / 3$ in units with $G=1=c$, where $M$ is the mass of the central body, $t$ the cosmic time and $r$ the physical distance from the center of mass.

The trajectory $(t(\tau), r(\tau), \theta(\tau), \phi(\tau))$ of a test particle parametrized by the proper time $\tau$, satisfies

$$
\begin{gathered}
A(r) \dot{t}^{2}-\frac{\dot{r}^{2}}{A(r)}-r^{2} \dot{\theta}^{2}-r^{2} \sin ^{2} \theta \dot{\phi}^{2}=1 \\
A(r) \dot{t}=\eta, \frac{d}{d \tau}\left(r^{2} \dot{\theta}\right)=r^{2} \sin \theta \cos \theta \dot{\phi}^{2}, r^{2} \sin ^{2} \theta \dot{\phi}=\lambda
\end{gathered}
$$

where $\eta$ and $\lambda$ are fixed by the initial conditions and the "dot" denotes differentiation with respect to $\tau$. For radial motions $(\lambda=0)$ and initial conditions $r\left(\tau_{0}\right)=r_{0}$ and $\dot{r}\left(\tau_{0}\right)=u_{0}$ we obtain

$$
\frac{1}{2} \dot{r}^{2}+U_{0}(r)=\mathcal{E}_{0}
$$

where $U_{0}(r)=-M / r-\Lambda r^{2} / 6$ and $\mathcal{E}_{0}=\left(\eta^{2}-1\right) / 2=u_{0}^{2} / 2+U_{0}\left(r_{0}\right)$.

The force acting on a unit test mass is given by $F=-d U_{0} / d r=-M / r^{2}+\Lambda r / 3$. It vanishes at the critical radius $r_{c}=(3 M / \Lambda)^{1 / 3}$ or, in physical units for

$$
r_{c} \equiv\left(\frac{3 G M}{\Lambda c^{2}}\right)^{1 / 3}
$$


while $F$ is repulsive (attractive) for $r>r_{c}\left(r<r_{c}\right)$. Thus, a test particle left from rest at $r_{0}>r_{c}$ will be accelerated away from the spherical mass $M$, dragged by the accelerated expansion of the universe. For $r_{0}<r_{c}$ it will fall on the mass $M$, while $r_{0}=r_{c}$ is an unstable equilibrium position.

\subsection{Spherical collapse of early-Universe perturbation}

The treatment of a single spherical mass inside otherwise empty de Sitter space raises the question: is the limit expressed by Eq. (3.4) only applicable at very late times in the evolution of a $\Lambda \mathrm{CDM}$ universe, where the accelerated expansion leads to a matter distribution approximating the idealized configuration of the previous section? In this section we show that this is not the case. If instead we consider the evolution of a spherical overdensity from the early universe (where its density contrast with the background universe is extremely low) until the time it reaches turnaround, we derive exactly the same limit.

The evolution of a spherical perturbation of any size and sign in $\Lambda \mathrm{CDM}$ cosmology is governed by the ratio of the Friedmann equations for the perturbation and the background universe $[34,35]$,

$$
\left(\frac{d a_{\mathrm{p}}}{d a}\right)^{2}=\frac{a_{\mathrm{p}}^{-1}+\omega a_{\mathrm{p}}^{2}-\kappa}{a^{-1}+\omega a^{2}}=\frac{a}{a_{\mathrm{p}}} \frac{\omega a_{\mathrm{p}}^{3}-\kappa a_{\mathrm{p}}+1}{\omega a^{3}+1},
$$

where $a$ is the scale factor of the Universe, $a_{p}$ is the scale factor of the perturbation, $\kappa$ characterizes the amplitude of the perturbation, and $\omega=\Omega_{\Lambda} / \Omega_{m}$ for the background Universe. The smallest $\kappa$ for which $\omega a_{\mathrm{p}}^{3}-\kappa a_{\mathrm{p}}+1=0$ has a real positive solution (i.e. $a_{p}$ turns around) is $\kappa_{\text {min,coll }}=3 \omega^{1 / 3} / 2^{2 / 3}$. The corresponding solution is the maximum turnaround radius, $a_{\mathrm{p}, \mathrm{ta} \text {, } \max }=(2 \omega)^{-1 / 3}=\left(4 \pi G \rho_{m} / \Lambda c^{2}\right)^{1 / 3}$. All other collapsing overdensities will have $a_{\mathrm{p}, \mathrm{ta}}<a_{\mathrm{p}, \mathrm{ta} \text {,max }}$. The physical radius of a perturbation is $R_{\mathrm{p}}=a_{\mathrm{p}}\left(3 M / 4 \pi \rho_{m}\right)^{1 / 3}$, where $\rho_{m}$ is the matter density of the background Universe. We thus obtain

$$
R_{\mathrm{p}, \mathrm{ta}, \max }=\left(\frac{4 \pi G \rho_{m}}{\Lambda c^{2}}\right)^{1 / 3}\left(\frac{3 M}{4 \pi \rho_{m}}\right)^{1 / 3}=\left(\frac{3 G M}{\Lambda c^{2}}\right)^{1 / 3}
$$

which is independent of cosmic time.

\subsection{Zero Acceleration Mass Shell}

Having shown quantitatively that the limit exists and is the same in both idealized configurations we considered in the previous sections, we can express it in a fashion that unifies conceptually the two approaches.

Ultimately, the upper bound to the turnaround radius separates two qualitatively different regions in spacetime. For smaller radii, any mass shell at rest will accelerate inwards. For larger radii, even a mass shell at rest will accelerate outwards, due to the dominating influence of vacuum energy. The maximum turnaround radius mass shell, therefore, must be a zeroacceleration one: it should define a sub-universe obeying its own Friedmann equation, the time derivative of which satisfies $\ddot{a_{p}}=0$ (where $\ddot{a_{p}}$ is the second time derivative of the scale factor of the perturbation) when the matter density inside it is equal to $3 M / 4 \pi R_{\mathrm{ta}, \max }^{3}$.

The time derivative of the Friedmann equation can be written as

$$
\ddot{a_{p}}=-4 \pi G a_{p}\left(\rho c^{2}+3 p\right) / 3 .
$$


Therefore, the no-acceleration condition then yields

$$
\rho c^{2}+3 p=0
$$

where in our case $\rho=\rho_{v}+\rho_{m}$. For a matter term heavily dominated by pressureless dark matter, the only source of pressure is vacuum energy. But vacuum energy (assuming it behaves as a cosmological constant) satisfies $\rho_{v} c^{2}=-p_{v}$. Equation (3.8) then becomes

$$
\rho_{m}=2 \rho_{v}
$$

or $3 M / 4 \pi R_{\mathrm{ta}, \max }^{3}=2 \Lambda c^{2} / 8 \pi G$ which yields the familiar by now result

$$
R_{\mathrm{ta}, \max }=\left(\frac{3 G M}{\Lambda c^{2}}\right)^{1 / 3}
$$

which is independent of cosmic time.

As it is obvious from Eq. (3.9), an alternative way to state the limit is in terms of the mean matter density within the turnaround radius, $\rho_{\mathrm{m} \text {,ta }}$, which, from Eq. (3.9), has to satisfy $\rho_{\mathrm{m}, \mathrm{ta}} \geq 2 \rho_{v}$. If the vacuum density within a spherical region is higher than one-half the matter density within that region at any given time, then the mass shell at the edge of that region has to be expanding, and it will continue to do so indefinitely at an accelerated rate.

For a cosmology satisfying $\Omega_{m}(a)+\Omega_{\Lambda}(a)=1$, (where $\Omega$ denotes a density in units of the critical density $\rho_{\text {crit }}(a)$ at a specific cosmic epoch labeled by the cosmic scale factor $a$ ), we can express this limit in terms of the density contrast of a non-expanding spherical region with respect to the background matter density $\rho_{m, b}, \delta_{\mathrm{ta}}=\left(\rho_{m, \mathrm{ta}}-\rho_{m, b}\right) / \rho_{m, b}$ :

$$
\delta_{\mathrm{ta}}=\frac{\rho_{\mathrm{m}, \mathrm{ta}}}{\rho_{m, b}}-1 \geq \frac{2 \rho_{v}}{\rho_{m, b}}-1=\frac{2 \Omega_{\Lambda}(a)}{\Omega_{m}(a)}-1=\frac{2}{\Omega_{m}(a)}-3 .
$$

For the present cosmic epoch, the best estimate for $\Omega_{m}$ is 0.315 [12] so inequality (3.11) yields $\delta \geq 3.35$ : although the limit is applicable to non-expanding structures, it is relevant for densities that are more than 100 times smaller than the virial overdensity at the present cosmic time [24].

It is also clear that if $\Omega_{m}(a)>2 / 3$ then inequality (3.11) is satisfied for any overdensity (positive density contrast), and therefore the bound can only be used as a cosmological discriminator at late times in the evolution of the Universe. The redshift at which $\Omega_{m}(a)=2 / 3$ can be found from

$$
\Omega_{m}(a)=\frac{\rho_{m, b, \text { present }} a^{-3}}{\rho_{\text {crit,present }}\left[\Omega_{\Lambda}+\Omega_{m} a^{-3}\right]}=\frac{\Omega_{m} a^{-3}}{\Omega_{\Lambda}+\Omega_{m} a^{-3}} .
$$

For cosmological parameters from [12], Eq. (3.12) gives $a=(1+z)^{-1}=0.613$, and therefore meaningful observational tests of the bound must focus at redshifts $<0.63$. That at early times the bound does not come into play is qualitatively expected: at high redshifts where $\Lambda$ is very subdominant, structure formation should proceed in a manner indistinguishable from the $\Omega_{m}=1$ cosmology, which predicts no bound.

\section{Testing the bound observationally}

The maximum turnaround radius is a robust, time-independent prediction of $\Lambda$ CDM cosmology. The only assumption that enters its derivation is that of spherical symmetry. This prediction states that, in a $\Lambda \mathrm{CDM}$ universe, there can never be a nonexpanding structure whose radius is greater than $\left(3 G M / \Lambda c^{2}\right)^{1 / 3}$, where $M$ is the structure's mass. In this section, we discuss how this prediction can be used as an observational test of various cosmological models. 


\subsection{What observations are necessary?}

Observational tests of the bound would aim to answer the question: is the turnaround radius of a given structure less than $\left(3 G M / \Lambda c^{2}\right)^{1 / 3}$ ? This requires independent measurements of the turnaround radius around a center of mass, and the total mass enclosed within said radius.

The turnaround radius around a center of mass is a well-defined, although not always easy to measure: it is the radius where the average radial velocity with respect to said center is zero, while it is positive for all radii larger than the turnaround radius. The last part of the definition is necessary to distinguish between the accretion boundary and the turnaround radius in structures that experience infall: the average radial velocity changes from zero to negative as the accretion boundary is crossed outwards, and from zero to positive as the turnaround radius is crossed outwards.

The turnaround radius can be determined by high-precision observations of the Hubble flow in cosmic structures that consist of at least several galaxies. Such observations have been successfully pursued for a number of local-universe structures (typically galaxy groups or nearby clusters, e.g. [41-46]). The uncertainty with which the turnaround radius (commonly referred to as the "zero-velocity surface") has been determined in these studies is between $10-30 \%$.

The mass within the turnaround radius is more difficult to measure. Estimates of the turnaround radius are typically used in combination with predictions of the spherical collapse model in the preferred cosmology at the time of each study to obtain an estimate of the structure mass. This approach clearly enforces by construction the validity of the bound we propose to test here, so a different way to estimate the mass is needed. Summing up the masses of the constituent galaxies [47], lensing studies, or X-ray spectroscopy studies [48] could provide such alternatives.

A problem with such independent methods of mass estimation is that they usually do not correspond to regions extending out to the turnaround radius. This is partly a matter of observational strategy (the possibility of using mass measurements out to the turnaround radius as a test of cosmology has not been recognized up to now); often however it is a matter of sensitivity: the X-ray brightness of galaxy clusters, for example, would in most cases be too low to detect at the turnaround radius.

The question then arises: would it be possible to use such estimates of mass - independent from the determination of the turnaround radius, but only "counting" mass out to a smaller radial boundary - as tests of the bound? The answer is yes. Such a mass estimate constitutes a lower limit (let us call it $M_{11}$ ) to the total structure mass (let us call it $M_{\text {tot }}$ ) out to the turnaround radius. If the measured turnaround radius of the structure does not exceed the bound corresponding to a mass $M_{11}$, then it is not going to exceed the bound corresponding to its true total mass $M_{\text {tot }}$ either, since the value of bound increases monotonically with mass. If, on the other hand, the bound corresponding to $M_{11}$ is exceeded, it is not guaranteed that the bound corresponding to $M_{\text {tot }}$ will also be exceeded. Such a structure can be labeled as a candidate for bound violation, and targeted, more sensitive follow-up observations can be undertaken to determine the mass $M_{\text {tot }}$ (preferably by more than one methods), to either confirm or reject a bound violation together with its cosmological implications.

Similarly, some information can be extracted from structures for which good mass estimates of their mass out to some radius exist, but for which a reliable estimate of their turnaround radius is not possible. The bound discussed here is an absolute upper limit for all non-expanding shells around a center of gravity in $\Lambda \mathrm{CDM}$ cosmology. For this reason, any mass shell not moving away 
from the structure center cannot have a radius larger than $\left(3 G M / \Lambda c^{2}\right)^{1 / 3}$. An upwards violation of this limit by a non-expanding shell internal to the turnaround radius is still a violation of $\Lambda \mathrm{CDM}$ cosmology. In contrast, a mass/radius measurement that obeys the bound for a shell internal to the turnaround radius is non-informative.

\subsection{How many structures need to be observed?}

A single high-confidence observation of the turnaround radius of a structure of known mass exceeding the bound would be sufficient to constrain $\Lambda \mathrm{CDM}$ cosmology. However, even if the cosmological model differs from $\Lambda \mathrm{CDM}$ in such a way that the bound does not hold, not every structure will have a turnaround radius larger than $\left(3 G M / \Lambda c^{2}\right)^{1 / 3}$. The reason is the statistical nature of structure formation: different regions (enclosing similar mass) start off with slightly different average overdensities in the early universe and will have reached a different stage in their evolution by a certain redshift. As a result, the more structures we observe (estimate their turnaround radius and enclosed mass), the higher the probability to identify structures exceeding the bound (if such a violation is allowed by the actual cosmology of our Universe).

The number of structures that need to be observed in order to achieve a certain probability of encountering a bound-exceeding object depends on the cosmological model - alternative to $\Lambda \mathrm{CDM}$ - that would be producing such violations. For example, if the perceived acceleration of the expansion of the Universe were a result of a local large-scale inhomogeneity rather than a cosmological constant, as suggested, e.g., by [49-52], then there should be no bound to the turnaround radius - even regions with very low overdensities in the early universe are allowed to turnaround and eventually collapse. Because in the standard $\Lambda$ CDM model the acceleration of the Universe has largely suppressed structure formation by the present cosmic epoch (and thus almost all structures should be close to their ultimate size by now, e.g. $[28,29]$ ), the observation of only a few $(\lesssim 10)$ mass/turnaround radius pairs should be adequate to identify bound-violating objects.

For other cosmological models that also predict bounds but of a different amplitude, the number of sources that need to be observed for a certain probability of exceeding the bound can be calculated on a case-by-case basis. In general, the looser the bound of the "true" cosmology, the higher the chance to identify a bound-violating structure with a fixed number of observations.

A specific example of a cosmological model resulting in a bound of different amplitude is that of dark energy having a general equation of state parameterized by $w=p / \rho<-1 / 3$. In this case, the maximum turnaround radius is [53]

$$
R_{\mathrm{ta}, \max }=\left(-\frac{3 M}{4(1+3 w) \pi \rho_{E}}\right)^{1 / 3}
$$

which gives the same result as Eq. (3.4) for $w=-1$. The bound is tighter the more negative $w$ is. As discussed in [53], existing data may already indicate violations of the bound for $w<-1$. Several structures potentially violating the bound for $w=-2.5$ (which is tighter than the "true" bound by about a factor of 2 , assuming that $\Lambda$ CDM is the "true" cosmology) are found among observations of fewer than 10 objects.

\subsection{Which structures should we be observing?}

Because the bound is independent of cosmic time, while structures continue to grow with time and approach their maximum possible size, the best cosmic epoch to test the bound observationally is the present one. 
In a standard hierarchical structure formation scenario, smaller structures form first (have a higher chance to begin with high overdensities in the early Universe), and are also the first to reach their maximum sizes. For this reason, the optimal mass range to target in order to possibly identify bound-violating structures would be the smallest masses for which the turnaround radius can be confidently determined. Assuming that the turnaround radius estimate would be done using the Hubble-flow analysis employed in past studies (e.g. [42]), then we need several galaxymembers within the target structure, so the natural class of targets would be groups of galaxies.

More accurate estimates of the range of masses that hold the maximum discriminating power (i.e. that are likely to have reached their maximum attainable mass at the present cosmic epoch while at the same time being large enough to allow confident determination of their turnaround radius) for various cosmological models can be produced through simulations and semi-analytical models.

\subsection{Can such observations produce "false positives"?}

If, then, $R$ or, equivalently, $\rho$ that violate the limit are measured, the discrepancy cannot be attributed to baryonic physics (which is only a very small perturbation at turnaround scales), complexities of the process of collapse and dynamical relaxation, or uncertainties in the way radii are defined. The only simplifying assumption entering its calculation is spherical symmetry, and accounting for deviations from sphericity is not expected to change this result significantly, as a mass shell at its moment of turnaround has not undergone any contraction yet. However, if the bound is to be used to confidently constrain the cosmological model, the magnitude of any non-sphericity effects has to be determined accurately.

The effect of non-sphericities on turnaround and the ultimate radii of cosmic structures has been discussed analytically by [33] and, to some extent, in cosmological simulations by [29]. In the latter work, it was found that, for realistic structures with mass between $10^{14}$ and $10^{16} \mathrm{M}_{\odot}$, the sphere of influence is no more than $30 \%$ larger than the what would be expected in spherical symmetry. Ideally, the problem should be addressed by analysis of cosmological simulations targeting the mass ranges of interest and using the same way to define the turnaround radius in simulations and observations of asymmetric structures.

\subsection{How accurate need the observations be?}

Assuming in the future an analysis tailored to the purposes of the test proposed here provides independent measurements for both mass and turnaround radius of a sample of structures large enough so as to include at least one structure with mass very close to its final mass (see §2), we give below a very simple estimate of the confidence at which various levels of violation of the bound can be established, given a certain accuracy for measurement of structure mass and radius.

Let us assume that a structure of final mass $M$ exceeds the bound by an amount $\Delta R$, i.e. its radius $R$ is equal to

$$
R=R_{\mathrm{ta}, \max }+\Delta R=\left(\frac{3 G M}{\Lambda c^{2}}\right)^{1 / 3}+\Delta R
$$

The question that we would like to answer is the following: for a given fractional violation of the bound, i.e. for a given value of $\lambda=\Delta R / R_{\mathrm{ta}, \max }$, what is the uncertainty in the measurements of $R$ and $M$ we can tolerate, to establish the violation of the bound at a given confidence level 
(number of $\sigma$ )? We will denote the number of $\sigma$ with $n$, the uncertainty in the measurement of radii with $\sigma_{R}$, and the uncertainty in the measurement of masses with $\sigma_{m}$. We measure $\Delta R$ by measuring $R$ directly, and by estimating $R_{\mathrm{ta} \text {,max }}$ indirectly, using a mass measurement: $\Delta R=R-R_{\mathrm{ta} \text {,max }}$. Simple error propagation implies that $\sigma_{\Delta R}^{2}=\sigma_{R}^{2}+\sigma_{R_{\mathrm{ta} \text {, max }}^{2}}$. Similarly, $\sigma_{R_{\mathrm{ta}, \max }}^{2}$ depends on the uncertainty in our mass measurement $\sigma_{R_{\mathrm{ta}, \max }} / R_{\mathrm{ta}, \max }=(1 / 3) \sigma_{m} / M$, where we have ignored the -much smaller- error entering through $\Lambda$. Combining these two results, we obtain

$$
\sigma_{\Delta R}^{2}=\sigma_{R}^{2}+\frac{1}{9} \sigma_{m}^{2} \frac{R_{\mathrm{ta}, \max }^{2}}{M^{2}}
$$

Using

$$
n=\frac{\Delta R}{\sigma_{\Delta R}}=\lambda \frac{R_{\mathrm{ta}, \max }}{\sigma_{\Delta R}}
$$

we can write Eq. (4.3) as

$$
\lambda^{2}=n^{2}\left(\frac{\sigma_{R}^{2}}{R_{\mathrm{ta}, \max }^{2}}+\frac{1}{9} \frac{\sigma_{m}^{2}}{M^{2}}\right) .
$$

Equation (4.5) implies that a few percent accuracy in the measurements of $R$ and $M$ allows us to establish a $10 \%$ bound violation at the $3 \sigma$ level, and a $6 \%$ violation at $2 \sigma$. If we were to simply increase our sample of structures with independently measured $R_{\mathrm{ta}}$ and $M$ without any improvement in measurement techniques (i.e. with uncertainties similar to the ones quoted for the few cases discussed above, $\sigma_{m} / M \sim 30 \%, \sigma_{R} / R \sim 5 \%$ ), for a structure close to its final mass we can establish a $30 \%$ bound violation at $3 \sigma$, and we can already detect a $1 \sigma$ tension between data and prediction for a $10 \%$ violation. An improvement in mass measurements would at this point have the largest impact in terms of updating observational techniques.

Regarding the effect of non-sphericities, if it can be simply represented as a multiplicative factor $f$ on the spherically symmetric result, i.e.

$$
R_{\mathrm{ta}, \mathrm{max}, \text { actual }}=f R_{\mathrm{ta}, \mathrm{max}, \text { spherical symmetry }}
$$

then the results above still hold, as long as $\lambda$ describes a fractional violation over the actual bound, including any non-sphericity corrections. As a result, an increase in accuracy with which deviations from spherical symmetry are accounted for can be best achieved with systematic analysis of ultimate turnaround radii as a function of mass in simulations, with the radii defined as closely as possible to the way they can be measured in observations; the observational accuracies required are still those quoted above.

\subsection{What can already existing data tell us?}

From the latest determination of the cosmological parameters by Planck [12], the present estimate of the matter density parameter is $\Omega_{m}=0.315 \pm 0.017$ so $\Omega_{\Lambda}=1-\Omega_{m}=0.685$. For this value of $\Omega_{\Lambda}$, the bound becomes $R_{\mathrm{ta}, \max }=11.2 \pm 0.1 \mathrm{Mpc}\left(M / 10^{15} M_{\odot}\right)^{1 / 3}$. In the discussion below, whenever the value of the Hubble parameter was needed to obtain a mass or radius in physical units for specific objects, we have used $H_{0}=67.3 \mathrm{~km} \mathrm{~s}^{-1} \mathrm{Mpc}^{-1}$ [12].

A few sanity checks yield comforting results. For the solar system, a central mass of $1 \mathrm{M}_{\odot}$ yields a bound of $\sim 100 \mathrm{pc}$, much greater than the solar system radius. The sun would be able to hold onto his own even if the hand of some angry deity were to cast the solar system in the middle of a cosmic void. For the Milky Way, assuming that the Leo I dwarf spheroidal at a 


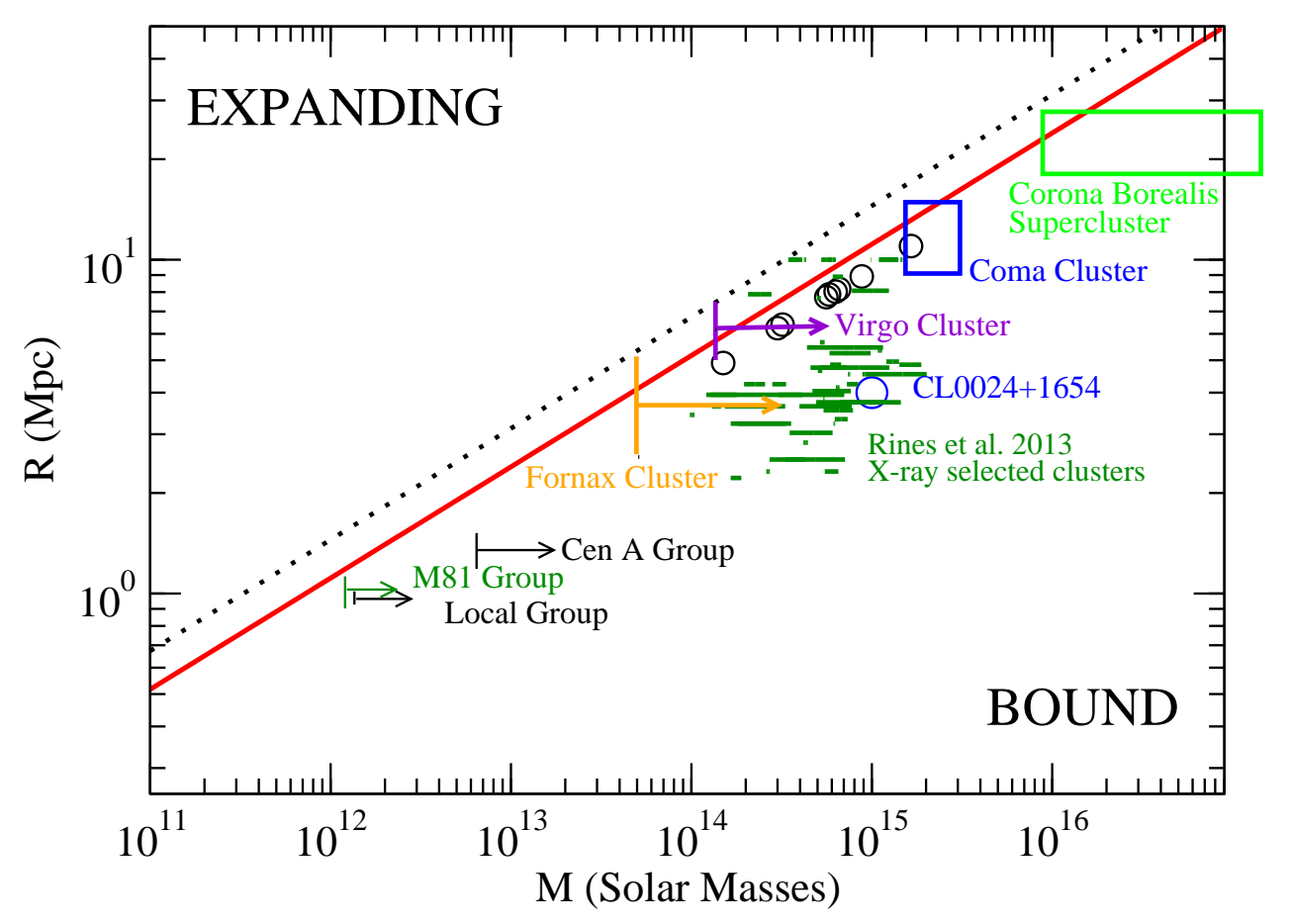

Figure 1. Data masses and turnaround radii for several local-Universe structures compared to the $\Lambda$ CDM turnaround radius bound (red line). The dotted line represents the location of the bound accounting for the estimated effect of non-sphericities. See text for details.

distance of $254 \pm 16 \mathrm{kpc}$ is bound to the Galaxy [54,55], $M=1-2.4 \times 10^{12} \mathrm{M}_{\odot}$ yielding a bound of $1.1-1.5 \mathrm{Mpc}$, much larger that the Leo I distance.

In Fig. 1 we have compiled data from the literature on the radii and masses of groups of galaxies, clusters of galaxies, and galaxy superclusters, and we plot them together with the turnaround radius bound: with the red solid line, we plot the spherically symmetric result, derived in this work; with the black dotted line we plot our estimate (using the simulation results of [29]) of the bound including the effect of deviations from spherical symmetry.

Because the analyses that resulted in this data were not tailored to the test we wish to perform here, in most cases we do not have independent estimates of the turnaround radius and the mass enclosed out to the turnaround radius. For example, [56] calculate mass profiles for clusters A119, A168, A496, A539, A576, A1367, A1656, A2199 and A194 using the caustic technique, and then use a constant-overdensity criterion to calculate the location of the turnaround radius. Although such data cannot be used to test the bound (since the radius and mass estimates are derived from each other), we plot this data with open black circles for illustration purposes in Fig. 1. As expected, they lie on a line parallel to that defined by the bound, as both the analysis of [56] and the bound correspond to a constant matter density. In contrast, we also plot in dark green a sample of 58 X-ray selected clusters from [57] that also had their mass measured using the caustic technique, out to a maximum radius where caustics were detected. This data populates almost an order of magnitude in radius for similar masses, as expected for a set of structures at different evolutionary stages (as clusters of galaxies are believed to be in the current cosmic epoch). Five of these structures (A1361, A1033, A1235, A646, A1366) exceed the 
spherically symmetric bound but not the estimated bound when non-sphericities are included; and two structures (A2055, A1918) reach the latter as well.

In other cases, the turnaround radius is directly obtained by examining the Hubble flow and the peculiar velocities around a given cosmic structure (e.g., [41-46]). These estimates are typically used to derive the enclosed mass based on spherical collapse model fitting. Clearly, this approach enforces by construction the validity of the bound we propose here to test, so these mass estimates cannot be used in combination with the turnaround radius measurements to test the bound. What we do instead is look for independent estimates of the mass of the structures in question. These generally do not estimate the mass out to the turnaround radius, but rather out to some smaller radial scale. These mass estimates then represent lower limits on the mass enclosed within the turnaround radius, and have been plotted as such in Fig. 1. We have included such measurements for:

- the Local Group (turnaround radius from [41]; mass estimate: lower limit on the sum of the masses of M31 and the Milky Way, as estimated by [58]);

- the M82 group of galaxies (turnaround radius from [59]; mass estimate: sum of virial masses of members [59]).

- the Cen A group of galaxies (turnaround radius from [43]; mass estimate: member orbital mass [43]);

- the Fornax cluster (turnaround radius from [45]; mass estimate: caustic technique within a smaller radius of $1.4 \mathrm{Mpc}[60])$;

- the Virgo cluster (turnaround radius from [46]; mass estimate: X-ray spectroscopic mass within smaller radius of $1.2 \mathrm{Mpc}$ [61]).

In Fig. 1 we also plot, with the open blue circle, data for the mass of CL0024+1654, obtained through lensing studies [62]. In this case, radius and mass match, but the radius is not the turnaround radius. The point does not exceed the bound so, as discussed in Section 4.1, such an observation is non-informative.

For the Coma cluster, we use data from the study of its infall region by [63]. The range of masses corresponds to the sum of the masses calculated by profile fitting within and outside the virial radius; the possible location of the turnaround radius is estimated from the location of the caustics in redshift space.

One of the two largest known structures in the local universe is the Corona Borealis supercluster (the second being the Shapley supercluster which, however, appears not to have reached turnaround at present [64], and the mass/size of which is therefore not constrained by the turnaround radius bound). Whether the Corona Borealis supercluster as a whole has reached turnaround at present is still a matter of debate [65]; however, the latest data and analysis of [66] indicate that this is likely the case, so we include data on its mass and radius in Fig. 1. The mass range results from estimates by [66] using a variety of different methods (including the virial theorem, the caustics technique, and orbital velocity/position fitting to the spherical collapse model). The turnaround radius these authors derive is not independent from their mass estimate (they use the spherical collapse model to estimate a turnaround radius from their mass estimate); instead, we use as lower and upper limits on the turnaround radius the distances from the center of the supercluster of the farthest member clusters that is collapsing towards the center, and the nearest member cluster that is receding from it, respectively. 
All of the structures in Fig. 1 are consistent within uncertainties with the maximum turnaround radius limit. However, there are also a number of structures that, within uncertainties, are also candidates for a possible limit violation (although none exceed our estimate of the bound when the effect of non-sphericities is included). Future observational or analysis efforts seeking to test the limit at higher confidence could potentially focus on these structures first.

Different radii plotted in Fig. 1 have different degrees of proximity to the exact definition of a turnaround radius as discussed in Section 4.1. The "zero velocity surface" of e.g. [41] is conceptually identical ("a surface which separates the overdensity against the homogeneous cosmic expansion", practically the surface that separates galaxies with positive and negative radial velocities compared to the group centroid). Even in this case, however, differences might arise due "real-world" effects (non-spherical structures, in which case, there is not enough information to reconstruct the full velocity field from the accessible line-of-sight velocity observations, and peculiar velocities) and the way this surface is determined in practice (e.g. lack of a sufficient number of objects close to the turnaround radius to determine the radius accurately). It is less clear how closely the "maximum radius" of [57] corresponds to the turnaround radius of a structure. The most direct way to assess such results and improve the comparison with theoretical expectations is by conducting mock observations using numerical simulations. In such simulations, the exact turnaround radius (as defined here) is known for every structure, and the values that would be derived by observations for each observable radius (zero velocity surface, maximum caustic radius etc) can also be estimated, yielding dependable, mass-dependent estimates of any systematic deviations.

The structures that are plotted on Fig. 1 were selected based on data availability rather than proximity to the limit; yet, all of them are close to the limit, indicating that structure growth is reaching its end at the current cosmic epoch. On the other hand, we do not see any strong violations of the limit among these $\sim 60$ structures (covering 4 orders of magnitude in mass) either. These two facts together are an important, local indication of dark energy at work.

All of the smaller structures (groups of galaxies) that we have examined lie under the limit even within errors. If hierarchical $\Lambda \mathrm{CDM}$ structure formation holds, this is most likely the effect of more accurate observations: in this scenario, it is the smallest structures that reach their limiting size first. Indeed, the fractional errors in the determinations of the turnaround radius in smaller structures are significantly smaller. If this is indeed the case, as observations of larger structures improve, these points will also move to the lower right of Fig. 1. This will be a strong argument in favor of $\Lambda \mathrm{CDM}$ cosmology and against inhomogeneous no- $\Lambda$ alternatives.

On the other hand, if more accurate observations of higher-mass structures confirm that these structures are closer to the limit, or even violating it, this will constitute evidence hard to reconcile with $\Lambda \mathrm{CDM}$.

\section{Discussion}

That essential to our existence structures (such as the solar system or, by chemical-evolution arguments, the Milky Way) do not lie close to the limit, prohibits the use of the limit to provide an "anthropic" explanation to the low value of the cosmological constant, consistent with the findings of [13]. However, it has not escaped our notice that a strong limit on the value of $\Lambda$ could be placed by requiring that measuring $\Lambda$ be feasible (see, e.g., [67].)

Alternative cosmological models have different predictions for the maximum turnaround radius, if a bound exists at all. For example, the dark energy equation of state will affect the 
value of the maximum (since no dark energy corresponds to no bound at all) [53]. MOND predictions would depend on the specifics of each theory, but they are in practice guaranteed to be different from $\Lambda \mathrm{CDM}$, while it is not obvious that following the three lines of reasoning presented here would even result to the same answer for a MOND-consistent gravity theory (Skordis, private communication). Although a detailed treatment of the bound in alternative cosmologies is beyond the scope of this letter, it is clear that the bound can act as a cosmology discriminator.

Even without improving the accuracy of mass and radius measurements, a larger sample of structures with independently observed turnaround radius and included mass could give at least first indications for bound violations down to $10 \%$. We therefore urge towards such efforts, which will challenge the limit and $\Lambda \mathrm{CDM}$ cosmology.

\section{Acknowledgments}

TNT would like to thank John Iliopoulos for useful discussions and his suggestion to make these notes public and C. Skordis for insightful comments on structure formation in the MOND framework. We thank Enea Romano and Dimitrios Tanoglidis for stimulating discussions, and an anonymous referee for constructive comments that improved this paper. This work was supported in part by European Union's Seventh Framework Programme under grant agreements (FP7-REGPOT-2012-2013-1) no 316165, PIF-GA-2011-300984, PCIG10-GA-2011-304001, PIRSESGA-2012-316788, the EU program "Thales" MIS 375734 and was also co-financed by the European Union (European Social Fund, ESF) and Greek national funds through the Operational Program "Education and Lifelong Learning" of the National Strategic Reference Framework (NSRF) under "Funding of proposals that have received a positive evaluation in the 3rd and 4th Call of ERC Grant Schemes" and under the "ARISTEIA" Action.

\section{References}

[1] Bergström, L. 2012, Annalen der Physik, 524, 479

[2] Bertone, G., Hooper, D., \& Silk, J. 2005, Phys. Rep., 405, 279

[3] Bradač, M., Allen, S. W., Treu, T., et al. 2008, ApJ, 687, 959

[4] Clowe, D., Markevitch, M., Bradač, M., et al. 2012, ApJ, 758, 128

[5] Markevitch, M., Gonzalez, A. H., Clowe, D., et al. 2004, ApJ, 606, 819

[6] Daylan, T., Finkbeiner, D. P., Hooper, D., et al. 2014, arXiv:1402.6703

[7] Bulbul, E., Markevitch, M., Foster, A., et al. 2014, arXiv:1402.2301

[8] Boyarsky, A., Ruchayskiy, O., Iakubovskyi, D., \& Franse, J. 2014, arXiv:1402.4119

[9] Famaey, B., \& McGaugh, S., 2012, Living Rev. Relativity, 15, 10

[10] Milgrom, M., 2010, PoS HRMS2010, 033

[11] Angus, G. W., Famaey, B., \& Diaferio, A. 2010, MNRAS, 402, 395

[12] Planck Collaboration, Ade, P. A. R., Aghanim, N., et al. 2013, arXiv:1303.5076

[13] Weinberg, S. 1987, Physical Review Letters, 59, 2607

[14] Famaey, B., \& McGaugh, S. 2013, Journal of Physics Conference Series, 437, 012001

[15] Brooks, A. M., Kuhlen, M., Zolotov, A., \& Hooper, D. 2013, ApJ, 765, 22 
[16] Arraki, K. S., Klypin, A., More, S., \& Trujillo-Gomez, S. 2014, MNRAS, 438, 1466

[17] Garrison-Kimmel, S., Rocha, M., Boylan-Kolchin, M., Bullock, J. S., \& Lally, J. 2013, MNRAS, 433, 3539

[18] Peter, A. H. G., \& Benson, A. J. 2010, PhysRevD, 82, 123521

[19] Vogelsberger, M., Zavala, J., \& Loeb, A. 2012, MNRAS, 423, 3740

[20] Lovell, M. R., Eke, V., Frenk, C. S., et al. 2012, MNRAS, 420, 2318

[21] Anderhalden, D., Schneider, A., Macciò, A. V., Diemand, J., \& Bertone, G. 2013, JCAP, 3, 14

[22] Peter, A. H. G., Rocha, M., Bullock, J. S., \& Kaplinghat, M. 2013, MNRAS, 430, 105

[23] Navarro, J. F., Frenk, C. S., \& White, S. D. M. 1996, ApJ, 462, 563

[24] Lahav, O., Lilje, P. B., Primack, J. R., \& Rees, M. J. 1991, MNRAS, 251, 128

[25] Tavio, H., Cuesta, A. J., Prada, F., Klypin, A. A., \& Sanchez-Conde, M. A. 2008, arXiv:0807.3027

[26] Cuesta, A. J., Prada, F., Klypin, A., \& Moles, M. 2008, MNRAS, 389, 385

[27] Shull, J. M. 2014, ApJ, 784, 142

[28] Nagamine, K., \& Loeb, A. 2003, NewA, 8, 439

[29] Busha, M. T., et al. 2003, ApJ, 596, 713

[30] Teerikorpi, P., Chernin, A. D., Karachentsev, I. D., \& Valtonen, M. J. 2008, A\&A, 483, 383

[31] Eingorn, M., \& Zhuk, A. 2012, JCAP, 9, 26

[32] Eingorn, M., Kudinova, A., \& Zhuk, A. 2013, JCAP, 4, 10;

[33] Barrow, J. D., \& Saich, P. 1993, MNRAS, 262, 717

[34] Eke, V. R., Cole, S., \& Frenk, C. S. 1996, MNRAS, 282, 263

[35] Pavlidou, V., \& Fields, B. D. 2005, PhysRevD, 71, 043510;

[36] Belli, S., Newman, A. B., \& Ellis, R. S. 2014, ApJ, 783, 117

[37] Noerdlinger, P. D., \& Petrosian, V. 1971, ApJ, 168, 1

[38] G.C. McVittie, Mon.Not.R.Astron.Soc. 933, 325 (1933)

[39] Chang Jun Gao, 2004, Class. Quant. Grav., 21, 4805, and earlier references therein

[40] Kaloper, N., Kleban, M., \& Martin, D. 2010, PhysRevD, 81, 104044

[41] Karachentsev, I. D., Sharina, M. E., Makarov, D. I., et al. 2002, A\&A, 389, 812

[42] Karachentsev, I. D., \& Kashibadze, O. G. 2006, Astrophysics, 49, 3

[43] Karachentsev, I. D., Tully, R. B., Dolphin, A., et al. 2007, AJ, 133, 504

[44] Karachentsev, I. D., \& Nasonova, O. G. 2010, MNRAS, 405, 1075

[45] Nasonova, O. G., de Freitas Pacheco, J. A., \& Karachentsev, I. D. 2011, A\&A, 532, A104

[46] Karachentsev, I. D., Tully, R. B., Wu, P.-F., Shaya, E. J., \& Dolphin, A. E. 2014, ApJ, 782, 4

[47] Li, Y.-S., \& White, S. D. M. 2008, MNRAS, 384, 1459

[48] Israel, H., Reiprich, T. H., Erben, T., et al. 2014, A\&A, 564, A129

[49] Kai, T., Kozaki, H., Nakao, K., Nambu, Y., \& Yoo, C. 2007, Progress of Theoretical Physics, 117, 229

[50] Romano, A. E. 2010, PhysRevD, 82, 123528 
[51] Mattsson, T. 2010, General Relativity and Gravitation, 42, 567

[52] Romano, A. E, \& Chen, P. 2011, JCAP, 10, 16

[53] Pavlidou, V., Tetradis, N., \& Tomaras, T. N. 2014, JCAP, 5, 17

[54] Boylan-Kolchin, M. et al. 2013, ApJ, 768, 140

[55] Bellazzini, M., Gennari, N., Ferraro, F. R., \& Sollima, A. 2004, MNRAS, 354, 708

[56] Rines, K., Geller, M. J., Kurtz, M. J., \& Diaferio, A. 2003, AJ, 126, 2152

[57] Rines, K., Geller, M. J., Diaferio, A., \& Kurtz, M. J. 2013, ApJ, 767, 15

[58] Gonzalez, R. E., Kravtsov, A. V., \& Gnedin, N. Y. 2013, arXiv:1312.2587

[59] Karachentsev, I. D., Dolphin, A. E., Geisler, D., et al. 2002, A\&A, 383, 125

[60] Drinkwater, M. J., Gregg, M. D., \& Colless, M. 2001, ApJL, 548, L139

[61] Urban, O., Werner, N., Simionescu, A., Allen, S. W., Böhringer, H. 2011, MNRAS, 414, 2101

[62] Kneib, J.-P., Hudelot, P., Ellis, R. S., et al. 2003, ApJ, 598, 804

[63] Rines, K., Geller, M. J., Kurtz, M. J., et al. 2001, ApJL, 561, L41

[64] Muñoz, J. A., \& Loeb, A. 2008, MNRAS, 391, 1341

[65] Batiste, M., \& Batuski, D. J. 2013, MNRAS, 436, 3331

[66] Pearson, D. W., Batiste, M., \& Batuski, D. J. 2014, MNRAS, 441, 1601

[67] Sivanandam, N. 2013, PhysRevD, 87, 083514 